\title{
REOXYGENATION ENHANCES TUMOUR NECROSIS FACTOR ALPHA- INDUCED DEGRADATION OF THE EXTRACELLULAR MATRIX PRODUCED BY CHONDROGENIC CELLS
}

\author{
B.D. Markway ${ }^{1}$, H. Cho ${ }^{1}$, D.E. Anderson ${ }^{1}$, P. Holden ${ }^{1}$, V. Ravi², C.B. Little ${ }^{2}$ and B. Johnstone ${ }^{1, *}$ \\ ${ }^{1}$ Department of Orthopaedics and Rehabilitation, Oregon Health \& Science University, Portland, OR, USA \\ ${ }^{2}$ Kolling Institute, University of Sydney, Northern Local Health District, St Leonard, NSW, Australia
}

\begin{abstract}
Mesenchymal stem cells (MSCs) have been considered as a potential source for cell-based therapies in arthritic diseases for both their chondrogenic and anti-inflammatory properties. Thus, we examined how MSC-based neocartilage responds to tumour necrosis factor alpha (TNF- $\alpha$ ) compared to articular chondrocyte (AC)-based neocartilage. Since oxygen tension is altered in arthritic joints, we also examined how increased oxygen tension influences this process. Monolayer-expanded healthy human ACs and bone marrow MSCs were cultured in chondrogenic medium in three-dimensional culture under hypoxia. They were then exposed to TNF- $\alpha$ under hypoxic or increased oxygen tension. We found no inherent anti-inflammatory advantage' of MSC-derived neocartilage as it pertains to the enzymes studied here: more degradative enzymes were upregulated by TNF- $\alpha$ in MSCs than in ACs, regardless of the oxygen tension. MSCs were also more sensitive to reoxygenation during TNF- $\alpha$ exposure, as indicated by increased proteoglycan loss, increased aggrecanase-generated metabolites, and further upregulation of the major aggrecanases, ADAMTS4 and ADAMTS5. There was also evidence of matrix metalloproteinase (MMP)-mediated aggrecan interglobular domain cleavage and type II collagen loss in response to TNF- $\alpha$ in both MSCs and ACs, but more MMPs were further upregulated by reoxygenation in MSCs than in ACs. Our study provides further evidence that consideration of oxygen tension is essential for studying cartilage degradation; for example, neocartilage produced from MSCs may be more sensitive to the negative effects of repeated hypoxia/reoxygenation events than $\mathrm{AC}$-derived neocartilage. Consideration of the differences in responses may be important for cell-based therapies and selection of adjunctive chondroprotective agents.
\end{abstract}

Keywords: Mesenchymal stem cells, chondrocytes, inflammation, hypoxia, chondrogenesis.

\author{
*Address for correspondence: \\ Dr Brian Johnstone \\ Department of Orthopaedics and Rehabilitation \\ Oregon Health \& Science University \\ 3181 SW Sam Jackson Park Rd, OP31 \\ Portland, OR, USA.
}

Telephone Number: $(+1)$ 503-494-9505

FAX Number: $(+1)$ 503-494-5050

E-mail: johnstob@ohsu.edu

\section{Introduction}

In the past decade there has been growing evidence that inflammatory cytokines play a role in the cartilage degeneration seen in traumatic joint injuries and osteoarthritis (OA). Pro-inflammatory cytokines have recently been found to be elevated in the synovial fluid from not only OA joints, but also those with symptomatic cartilage defects (Tsuchida et al., 2012; Tsuchida et al., 2014). The recent study comparing synovial fluid and cartilage from healthy and OA joints found no significant difference in tumour necrosis factor- $\alpha$ (TNF- $\alpha$ ) levels (Tsuchida et al., 2014), but TNF- $\alpha$ has previously been reported to be significantly higher in synovial fluid of OA patients compared to those with inflammatory arthropathies (Westacott et al., 1990). While this study only examined synovial fluid from two healthy patients, and found TNF- $\alpha$ levels in them comparable to their highest OA specimens, a follow-up study showed that cultured synovial explants from OA patients produced significantly more TNF- $\alpha$ than those from non-arthritic subjects (Westacott et $a l ., 2000$ ). Interleukin-6 (IL-6) and TNF- $\alpha$ have been reported to be increased in patients who had undergone meniscectomies (Larsson et al., 2015) and in patients with ruptured anterior cruciate ligaments (Higuchi et al., 2006), both non-cartilage events that nonetheless predispose individuals to developing OA. Pro-inflammatory cytokines such as these can contribute to cartilage degeneration by stimulating aggrecanases and matrix metalloproteinases (MMPs) (Buttle et al., 1997; Flannery et al., 2000; Hughes et al., 1998; Little et al., 1999; Little et al., 2002). Repair strategies for focal cartilage defects need to consider this aspect of the injured joint environment. Due to their multipotency and anti-inflammatory potential, mesenchymal stem cells (MSCs) are a promising source for cell-based repair of injured tissue such as articular cartilage. In various injury models, MSCs have been suggested to aid in repair by secreting anti-inflammatory factors such as IL-1 receptor antagonist (IL-1ra) and TNF- $\alpha$ stimulated gene/protein 6 (TSG-6) (reviewed by Prockop and $\mathrm{Oh}, 2012$ ). However, IL-1 $\beta$ and TNF- $\alpha$ have both been shown to inhibit MSC chondrogenesis (Wehling et al., 2009 ) and IL-1 $\beta$ has been shown to cause aggrecanolysis in MSC-derived neocartilage (Boeuf et al., 2012). It remains unclear whether MSC-derived neocartilage has any anti-inflammatory advantage, reflected in less matrix degradation, compared with that derived from isolated articular chondrocytes.

Evaluating whether the origin of neocartilage constructs influences their ability to resist inflammatory factor-mediated degradation may be useful to inform 
future directions for tissue repair strategies. However, the conditions under which this is evaluated must include consideration of oxygen tension, which is acknowledged to be altered in injured joints, and which has a complex relationship with the activity of inflammatory factors. One theory regarding a mechanism for how alterations in oxygen tension may contribute to joint pathogenesis is that of persistent hypoxia-reperfusion injuries (Blake et al., 1989; Mapp et al., 1995; Vos et al., 2012). According to this theory, due to decreased capillary density in the inflamed superficial region of the synovium and high intra-articular pressure, normal mechanical loading leads to a reduction in oxygen delivery to cartilage. When loading is decreased reperfusion occurs and the sudden increase in oxygen delivery generates reactive oxygen species. Alternatively, it has also been proposed that a supraphysiological oxygen tension may exist in late stage OA when severe fibrillation of the cartilage exists (Martin et al., 2004), though there is also evidence that joints affected by trauma have elevated oxygen compared to diseased joints (Lund-Olesen, 1970). While the exact role of changes in oxygen tension during disease and injury remain unclear, it is evident that alterations do occur under a variety of joint pathologies.

Owing to the avascular nature of articular cartilage, chondrocytes exist in an oxygen environment much lower than that of the atmospheric levels in which isolated cells are usually studied (Brighton and Heppenstall, 1971; Silver, 1975; Zhou et al., 2004). It is well-established that low oxygen ("hypoxic") culture increases expression of cartilage matrix genes in human chondrocytes and enhances chondrogenesis of MSCs from various species (Kanichai et al., 2008; Lafont et al., 2008; Lafont et al., 2007; Markway et al., 2013; Markway et al., 2010; Robins et al., 2005). It has also been shown that hypoxic culture of healthy human chondrocytes decreases expression of catabolic enzymes (Markway et al., 2013; Strobel et al., 2010; Thoms et al., 2013), and that following formation of neocartilage under low oxygen, simply increasing oxygen tension leads to increased MMP expression and matrix degradation (Strobel et al., 2010). TNF- $\alpha$ and IL-1 $\beta$ have been reported to increase hypoxia inducible factor-1alpha (HIF-1 $\alpha$ ) expression in both healthy and OA chondrocytes (Clerigues et al., 2013; Coimbra et al., 2004) and EPAS1 (the HIF-2 $\alpha$ gene) in human and mouse chondrogenic cell lines (Saito et al., 2010). HIF-1 $\alpha$ and HIF-2 $\alpha$ have both been reported to be expressed more prominently in the synovium of diseased joints (Giatromanolaki et al., 2003; Hollander et al., 2001). Additionally, a number of groups have reported either increased HIF-1 $\alpha$ (Bohensky et al., 2009; Yudoh et al., 2005) or increased HIF-2 $\alpha$ (Saito et al., 2010; Yang et al., 2010) in osteoarthritic cartilage or chondrocytes, and several genes of catabolic enzymes associated with arthritis have been described as direct HIF-2 $\alpha$ targets (Hashimoto et al., 2013; Saito et al., 2010; Yang et al., 2010). Furthermore, it has been reported that IL-1 $\beta$-induced DNA binding of nuclear factor- $\kappa \mathrm{B}$ (NF$\kappa \mathrm{B})$ and activator protein 1 (AP-1), which can activate oxygen-independent HIF signalling, is enhanced in bovine chondrocytes cultured in hypoxia and those cultured in hypoxia followed by reoxygenation as compared with those in constant high oxygen (Martin et al., 2004). More recently it was reported that IL- $1 \beta$-induced MMP activity was lower in human OA chondrocytes, but increased in healthy chondrocytes, when maintained in hypoxia (Clerigues et al., 2013). Clearly there is a complex relationship between oxygen tension, inflammatory factors and their effects on chondrogenic cells. Therefore, we sought to evaluate how changes in oxygen environment affect neocartilage constructs and how TNF- $\alpha$-induced degradation of cartilage matrix is affected by oxygen tension. We used both articular chondrocyte (AC)- and MSC-derived neocartilage to examine whether the purported anti-inflammatory potential of MSCs imparted benefits to neocartilage derived from these cells.

\section{Materials and Methods}

\section{Isolation and expansion of human bone marrow MSCs and human ACs}

MSCs were isolated from iliac crest bone marrow aspirates ( $n=4$ donors) and expanded in monolayer culture as described previously (Johnstone et al., 1998; Yoo et al., 1998). Briefly, bone marrow aspirates obtained from consenting donors (IRB approved) were fractionated on a Percoll density gradient and plated at $160,000 \mathrm{cells} / \mathrm{cm}^{2}$ in Dulbecco's modified Eagle's medium (DMEM) with $10 \%$ foetal bovine serum (FBS). Adherent cells were cultured at $37{ }^{\circ} \mathrm{C}, 5 \% \mathrm{CO}_{2}$ (atmospheric oxygen) with medium changes every 3-4 d until confluent, at which point expansion medium was supplemented with $10 \mathrm{ng} / \mathrm{mL}$ basic fibroblast growth factor (FGF-2; Peprotech, Rocky Hill, NJ, USA). The MSCs used in this study were all confirmed to have high chondrogenic ability, accumulating at least as much sulphated glycosaminoglycan (sGAG) in pellet cultures under standard pellet culture conditions as our average ACs (data not shown).

ACs were isolated from normal cadaver femoral condyles that had been harvested within a few hours of death ( $n=5$ donors). Human tissue was transferred to Oregon Health \& Science University in a proprietary storage solution and obtained by the authors as discard tissue without patient identifiers. Oregon Health \& Science University Institutional Review board approved the study as exempt from needing consent. Cartilage was dissected from the condyles and finely minced before enzymatic digestion. Cartilage digestion was initiated with $1 \%$ protease from Streptomyces griseus (wt/vol) in low-glucose DMEM (Life Technologies, Grand Island, NY, USA) supplemented with $1 \%$ penicillin-streptomycin $(\mathrm{P} / \mathrm{S})$. After $1 \mathrm{~h}$ at $37^{\circ} \mathrm{C}$, protease was removed and replaced with $1300 \mathrm{U} / \mathrm{mL}$ collagenase II (Worthington Biochemical, Lakewood, NJ, USA) in DMEM $+\mathrm{P} / \mathrm{S}$ for $3 \mathrm{~h}$ at $37^{\circ} \mathrm{C}$. The cell suspension was passed through a $40-\mu \mathrm{m}$ cell strainer, centrifuged at $500 \times g$ for $5 \mathrm{~min}$, and the cells resuspended in low-glucose DMEM supplemented with $10 \% \mathrm{FBS}$ and $1 \% \mathrm{P} / \mathrm{S}$. Chondrocytes were plated at 7,000 cells $/ \mathrm{cm}^{2}$ and expanded in monolayer culture at $37{ }^{\circ} \mathrm{C}, 5 \% \mathrm{CO}_{2}$ (in atmospheric oxygen) with medium changes every 3-4 d. 


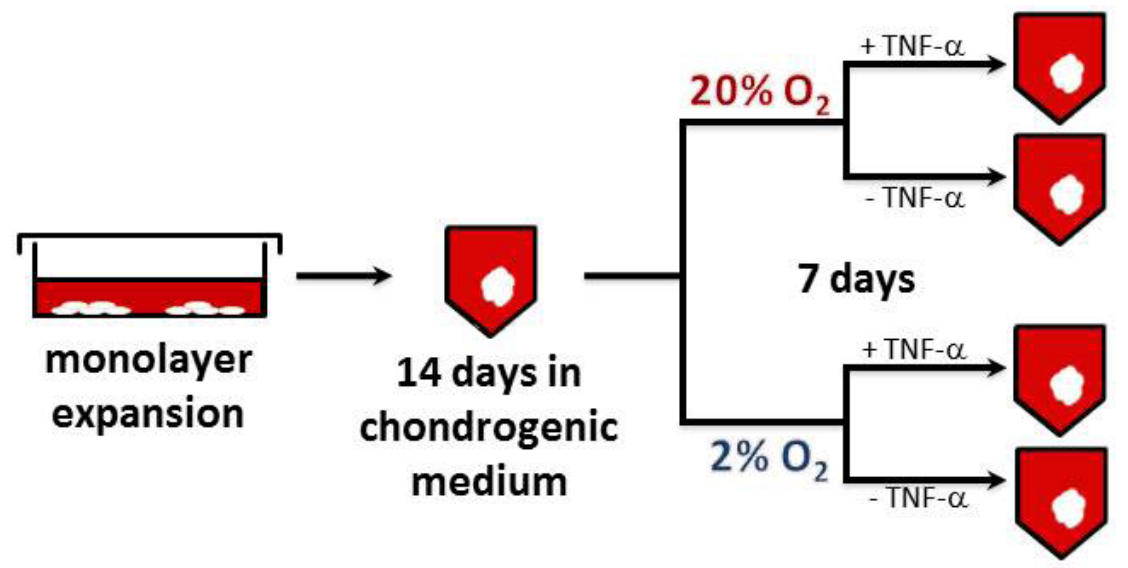

Fig. 1. Schematic of study design. MSCs and ACs were expanded in monolayer cultures in a standard tissue culture incubator $\left(20 \% \mathrm{O}_{2}\right)$ and then differentiated or redifferentiated, respectively, for 2 weeks in defined chondrogenic medium in hypoxia $\left(2 \% \mathrm{O}_{2}\right)$. At the end of 2 weeks, medium was changed to chondrogenic induction medium without TGF- $\beta 1$ and dexamethasone and with or without TNF- $\alpha(1 \mathrm{ng} / \mathrm{mL})$. At this point, pellets were either maintained in hypoxia or reoxygenated by moving them to a standard tissue culture incubator $\left(20 \% \mathrm{O}_{2}\right)$ and maintained for an additional week with medium changes every 2-3 $\mathrm{d}$.

\section{Pellet culture}

MSCs and ACs were pelleted between second and fourth passage with serum-free chondrogenic induction medium consisting of high-glucose DMEM (Gibco, Carlsbad, CA, USA) containing $10 \mathrm{ng} / \mathrm{mL}$ transforming growth factor- $\beta 1$ (TGF- $\beta 1$, Peprotech), $10^{-7} \mathrm{M}$ dexamethasone, $37.5 \mu \mathrm{g} / \mathrm{mL}$ ascorbic acid 2-phosphate, $1 \mathrm{mM}$ sodium pyruvate, $40 \mu \mathrm{g} /$ mL L-proline, 1X ITS+ (BD Biosciences, Franklin Lakes, NJ, USA), and $1 \% \mathrm{P} / \mathrm{S}$. Scaled down pellet cultures were set up by centrifuging $5 \times 10^{4}$ cells at $500 \times g$ in $240 \mu \mathrm{L}$ of medium in polypropylene V-bottom 96 -well plates (Nunc, Rochester, NY, USA) and maintained in a hypoxic chamber (Biospherix, Parish, NY, USA) set at $2 \%$ oxygen, $5 \% \mathrm{CO}_{2}$ (Markway et al., 2013; Markway et al., 2015). Medium was changed every 2-3 d while keeping the cells at $2 \%$ oxygen using a glovebox (Biospherix). At the end of 2 weeks of differentiation of MSCs or redifferentiation of ACs, medium was changed to chondrogenic induction medium without TGF- $\beta 1$ and dexamethasone and with or without TNF- $\alpha(1 \mathrm{ng} / \mathrm{mL})$. At this point, pellets were either maintained in hypoxia or reoxygenated by moving them to a standard tissue culture incubator and maintained for an additional week with medium changes every 2-3 d (Fig. 1). Although $\mathrm{CO}_{2}$ displacement actually lowers the oxygen level, hereafter we refer to the standard condition as $20 \%$ oxygen according to tissue culture convention, and we refer to a return to $20 \%$ oxygen as reoxygenation. Though $2 \%$ oxygen would more accurately be termed physiological, according to the convention of literature on the topic we have referred to this condition as hypoxia.

\section{Biochemical assays}

Biochemical assays were all conducted on at least triplicate pellets. Pellets were rinsed with phosphate-buffered saline (PBS) and digested overnight at $60{ }^{\circ} \mathrm{C}$ in $4 \mathrm{U} / \mathrm{mL}$ papain (Sigma, St. Louis, MO, USA) in PBS containing $6 \mathrm{mM} \mathrm{Na}$-EDTA and $6 \mathrm{mM}$ L-cysteine (papain buffer; pH 6.0). DNA content of all papain-digested pellets was quantified using $2 \mu \mathrm{g} / \mathrm{mL}$ Hoechst dye with calf thymus DNA diluted in papain buffer used to prepare standard curves. The sGAG content of pellets was quantified using 1,9-dimethymethylene blue (DMMB) dye ( $\mathrm{pH}$ 3.0). Shark chondroitin sulphate diluted in papain buffer was used to prepare standard curves.

RNA isolation and real-time quantitative polymerase chain reaction (qPCR)

RNEasy Mini Kit (Qiagen, Hilden, Germany) was used to collect total RNA from 4-6 replicate pellets of each condition for $n=4$ independent donors for each cell type. Pellets were snap-frozen in liquid nitrogen and crushed, then immediately lysed with RLT lysis buffer containing $40 \mathrm{mM}$ dithiothreitol (DTT). RNA isolation then proceeded as per the manufacturer's instructions.

RNA samples $(250 \mathrm{ng})$ were reverse transcribed using qScript cDNA SuperMix (Quanta Biosciences, Gaithersburg, MD, USA) as per the manufacturer's instructions. qPCR analysis was performed using a Bio-Rad MyiQ iCycler with Quanta PerfeCta qPCR FastMix (Quanta Biosciences) and TaqMan assays (Life Technologies) for MMP1 (Hs00899658_m1), MMP2 (Hs00234422_m1), MMP3 (Hs00968305_m1), MMP9 (Hs00234579_m1), MMP10 (Hs00233987m1), MMP12 (Hs00899662_m1), MMP13 (Hs00233992 m1), ADAMTS4 (Hs00192708_m1), and ADAMTS $\overline{5}$ (Hs00199841 m1). The cycling parameters were $45^{\circ} \mathrm{C}$ for $2 \mathrm{~min}, 95^{\circ} \mathrm{C}$ for $1 \mathrm{~min}$, and then $95^{\circ} \mathrm{C}$ for $5 \mathrm{~s}$ and $60^{\circ} \mathrm{C}$ for $30 \mathrm{~s}$ for a total of 40 cycles. Results were analysed using the $2^{-\Delta \mathrm{Ct}}$ method relative to the housekeeping gene 18S (Hs99999901_s1), which was deemed suitably stable for both cell types under the conditions described.

\section{Immunohistochemical analysis}

Pellets were fixed in $10 \%$ neutral buffered formalin, embedded in paraffin and sectioned onto slides. Representative samples of $7-\mu \mathrm{m}$ sections were stained with 
toluidine blue to visualise sulphated proteoglycans at points before (day 14) and after TNF- $\alpha$ treatment and oxygen alterations (day 21). Serial sections were deparaffinised in xylene, rehydrated through graded alcohols, and pretreated with (i) protease-free chondroitinase ABC $(0.1 \mathrm{U} /$ $\mathrm{mL}$; Sigma-Aldrich, St. Louis, MO, USA) and 0.1 U/ $\mathrm{mL}$ of keratanase I (Sapphire Biosciences, Alexandria, Australia) for NITEGE and DIPEN neoepitopes or (ii) bovine testicular hyaluronidase $(1000 \mathrm{U} / \mathrm{mL}$; SigmaAldrich) for aggrecan G1 domain and collagen type II. The following primary antibodies in antibody diluent (S0809, Dako, Glostrup, Denmark) were incubated overnight at $4{ }^{\circ} \mathrm{C}$ : aggrecan $\mathrm{G} 1$ domain $(1.5 \mu \mathrm{g} / \mathrm{mL}$; affinity-purified polyclonal antisera; provided by Prof John Mort, McGill University, Montreal, Canada), ADAMTS-generated aggrecan neoepitope NITEGE $(0.55 \mu \mathrm{g} / \mathrm{mL} ; \mathrm{mAb}$ Agg-C1; provided by Dr. Carl Flannery, Pfizer Inc, Cambridge, MA, USA), MMP-generated aggrecan neoepitope DIPEN $(0.17 \mu \mathrm{g} / \mathrm{mL}$; affinity-purified polyclonal antisera; provided by Prof Amanda Fosang, University of Melbourne, Melbourne, Australia), and collagen type II ( $5 \mu \mathrm{g} / \mathrm{mL} ; \mathrm{mAb}$ \#6317, MP Biomedicals, Santa Ana, CA, USA). Equivalent concentrations of species-matched immunoglobulins on identically treated sections were used as negative controls. After washing in Tris-buffered saline with Tween 20 (TBST) (Dako K800) slides were incubated with appropriate secondary antibody (EnVision+ rabbit (Dako K4003) or mouse (Dako K4001) horse-radish-peroxidase) for $30 \mathrm{~min}$ at room temperature. Colour was developed with NovaRED ${ }^{\mathrm{TM}}$ (SK-4800, VectorLabs, Burlingame, CA, USA) for $15 \mathrm{~min}$ at room temperature, and sections were then counter-stained with Mayer's haematoxylin with Scott's blueing solution.

\section{Western blotting}

Supernatant from the week of TNF- $\alpha$ exposure (days 14-21) was collected for each condition and samples from three biological replicates were pooled. For these samples ITS (Sigma), which does not include bovine serum albumin, was used for the week of TNF- $\alpha$ exposure in place of ITS+. sGAGs were quantified as described above and supernatant containing $30 \mu \mathrm{g}$ of sGAGs for each condition were concentrated using Amicon Ultra-4 10,000 kDa MWCO centrifugal filter units (Millipore, Billerica, MA, USA). Concentrated supernatants were precipitated with 9 volumes of cold ethanol, dried, resuspended in $0.1 \mathrm{M}$ Tris acetate buffer ( $\mathrm{pH}$ 6.5), and deglycosylated overnight at $37^{\circ} \mathrm{C}$ with $0.01 \mathrm{U}$ chondroitinase $\mathrm{ABC}, 0.01 \mathrm{U}$ keratanase, and 0.0001 U keratanase II (Seikagaku Corporation, Tokyo, Japan). Samples were then dialysed against ultrapure (Milli-Q) water at $4{ }^{\circ} \mathrm{C}$ overnight and freeze dried. Samples were separated electrophoretically under reducing conditions on $10 \%$ Bis-Tris gels (Invitrogen, Melbourne, Australia), transferred to nitrocellulose membranes (Invitrogen), blocked with $5 \%$ (w/v) bovine serum albumin (BSA; Sigma-Aldrich) and immunoblotted with a 1:100 dilution of monoclonal antibodies BC-3 (anti-ARGS) or BC-14 (anti-FFGV) (gift from Prof. B. Caterson, Cardiff University, Cardiff, UK) overnight as described previously (Little et al., 1999).

\section{Zymography}

Zymography was performed on either $10 \%$ SDS-PAGE gels containing $10 \mathrm{mg} / \mathrm{mL}$ gelatine or $12 \%$ gels containing $10 \mathrm{mg} / \mathrm{mL}$ casein. Medium samples collected at day 21 (day 7 of TNF- $\alpha$ treatment) were used for each condition. Samples were prepared in Laemmeli sample buffer without reducing agents or boiling, and $20 \mu \mathrm{L}$ of this was used for zymography. Samples were run alongside PageRuler Plus marker (Thermo Fisher Scientific, Cambridge, MA, USA) and then gels were incubated in renaturing buffer $(2.7 \%$ Triton-X100) for $2 \times 30 \mathrm{~min}$ before equilibrating in developing buffer $\left(50 \mathrm{mM}\right.$ Tris base, $5 \mathrm{mM} \mathrm{CaCl}_{2}, 40 \mathrm{mM}$ $\mathrm{HCl}, 200 \mathrm{mM} \mathrm{NaCl}, 0.02 \%$ (w/v) Brij-35) for $30 \mathrm{~min}$ at room temperature. The developing buffer was then changed and the gels were incubated at $37^{\circ} \mathrm{C}$ overnight. Developed zymograms were stained with Coomassie blue.

\section{Statistical analysis}

SigmaPlot 11.0 was used to assess statistical significance, which was defined as $p<0.05$. Four to five donors were used for each cell type and mRNA levels and sGAGs measured for each donor individually for quantitative assays. The number of donors analysed for each comparison is indicated in figure legends. Normality was evaluated using the Shapiro-Wilk test. Repeated measures ANOVA with Student-Newman-Keuls post hoc tests were used to compare means of multiple groups.

\section{Results}

\section{Oxygen-dependent effects of TNF- $\alpha$ on proteoglycan loss}

MSCs and ACs had distinct responses to the removal of the chondrogenic factors TGF- $\beta 1$ and dexamethasone as evidenced by the results from day 21 controls (Fig. 2a). Without these factors, MSCs continued to deposit sGAGs in the matrix as seen by the significantly higher amounts 1 week following withdrawal. In contrast, the matrix of ACs contained less sGAGs at day 21 if these factors were removed. The addition of TNF- $\alpha$ led to a significant net loss in sGAG content in reoxygenated MSC pellets and in AC pellets, regardless of whether they were maintained in hypoxia or reoxygenated (Fig. 2a). Reoxygenation significantly enhanced the TNF- $\alpha$-induced net loss of sGAGs in MSCs (3.4 \pm 1.1 fold). To visualise the loss of proteoglycans we stained pellets with toluidine blue and probed for aggrecan G1 domains. Changes in toluidine blue staining of the pellets were consistent with the quantitative sGAG data (Fig. 2b). Immunostaining for aggrecan G1 (Fig. 2c) suggested that loss of proteoglycan in TNF- $\alpha$ treated MSC and AC pellets could in part be explained by depletion of the entire aggrecan core-protein, particularly when the cells were reoxygenated.

\section{Oxygen-dependent effects of TNF- $\alpha$ on aggrecanase expression and activity}

In measuring changes in gene expression mediated by TNF- $\alpha$ and reoxygenation, we first evaluated the major aggrecanases responsible for aggrecan degradation in 
(a)

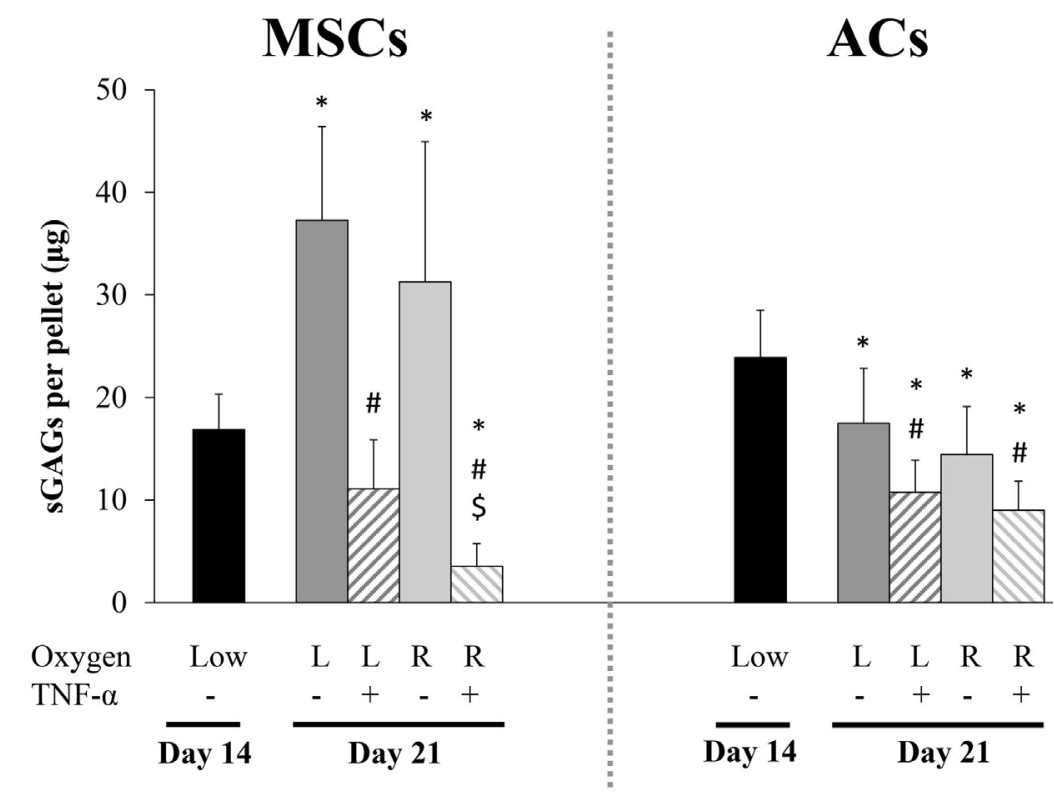

Fig. 2. Loss of proteoglycans

(b) in $\mathrm{MSC}$ - and $\mathrm{AC}$-derived neocartilage following TNF- $\alpha$ exposure and reoxygenation. (a) Proteoglycan content was quantified by measuring sGAGs in duplicate or triplicate pellets from $n=4$ (MSC) and $n=5$ (AC) individual donors at day $14\left(\mathrm{~d} 14: 2 \% \mathrm{O}_{2}\right.$ culture, pre-TNF- $\alpha$ exposure) and at day $21 \pm$ TNF- $\alpha$ with or without changes in oxygen. $\mathrm{L}=$ low oxygen $\left(2 \% \mathrm{O}_{2}\right), \mathrm{R}=$ reoxygenation $\left(20 \% \mathrm{O}_{2}\right)$. Values are the mean $\mu \mathrm{g}$ of sGAGs per pellet and error bars represent one standard deviation. Statistical significance was determined by repeated measures ANOVA with Student-Newman-Keuls post hoc tests; $p<0.05$ vs. d14 $\left.{ }^{*}\right)$, vs. matched oxygen level without TNF- $\alpha$ (\#), vs. low oxygen with TNF- $\alpha$ (\$). Loss of proteoglycans was visualised by (b) Toluidine blue staining and by (c) immunohistochemical staining for aggrecan G1 domain. Pellets of each experimental condition from one representative donor are shown. Scale bars $=200 \mu \mathrm{m}$.

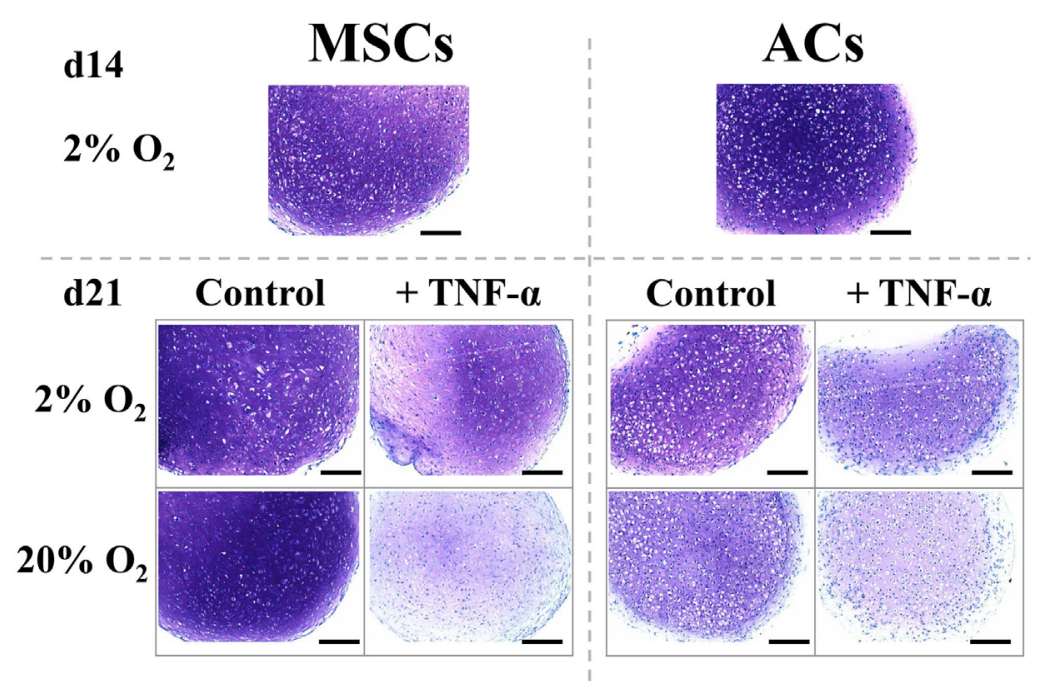

(c)

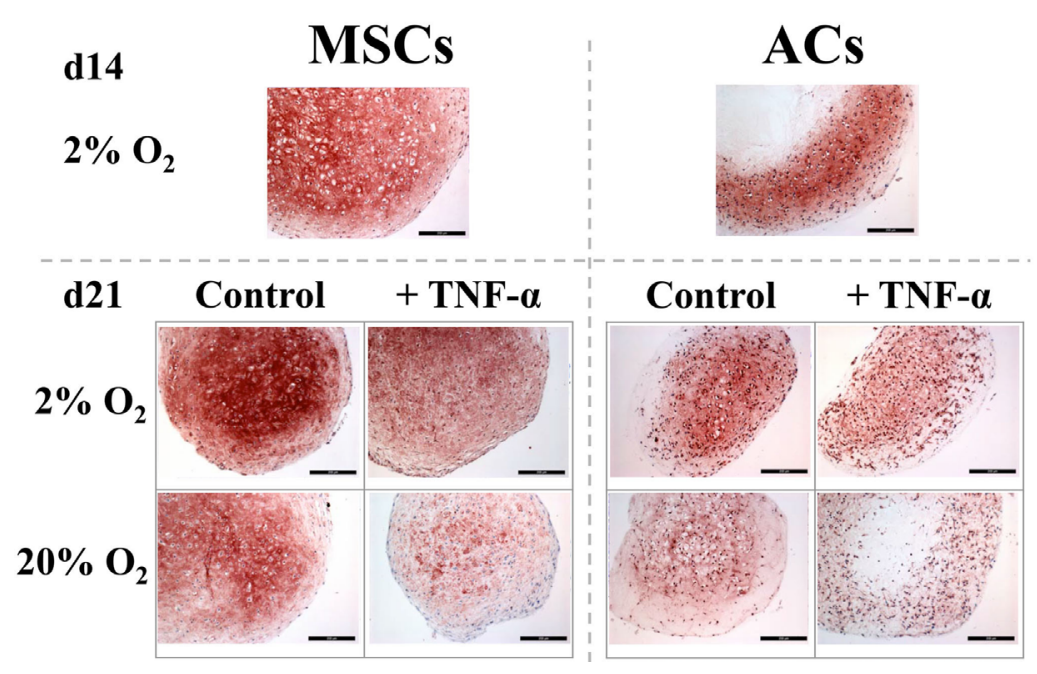


(a)

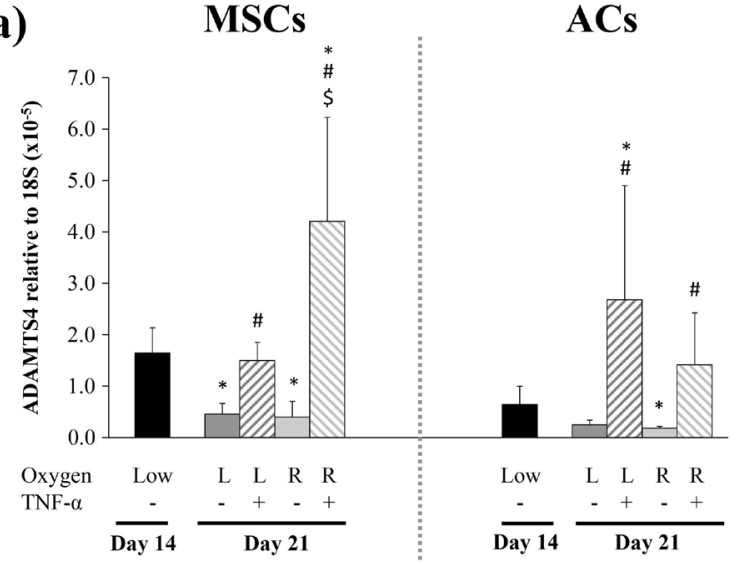

(c)

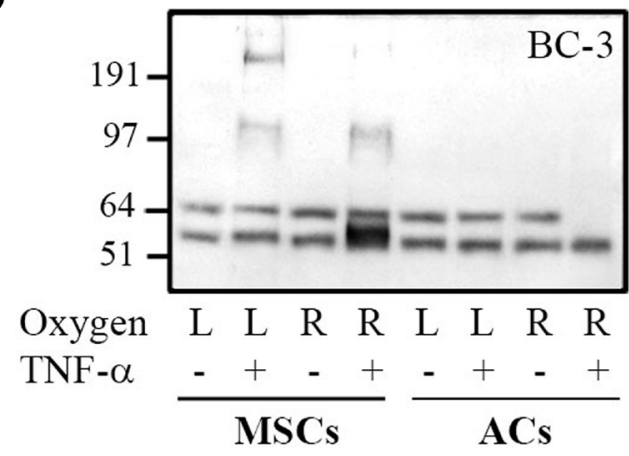

(b)

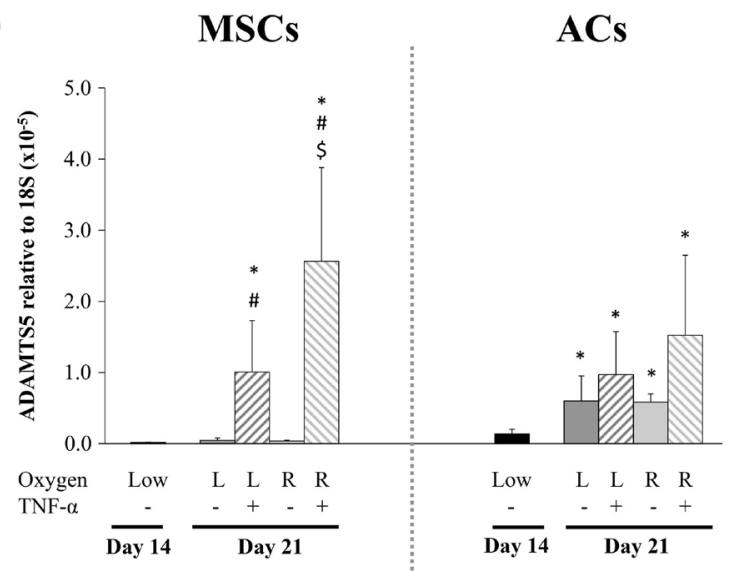

(d)

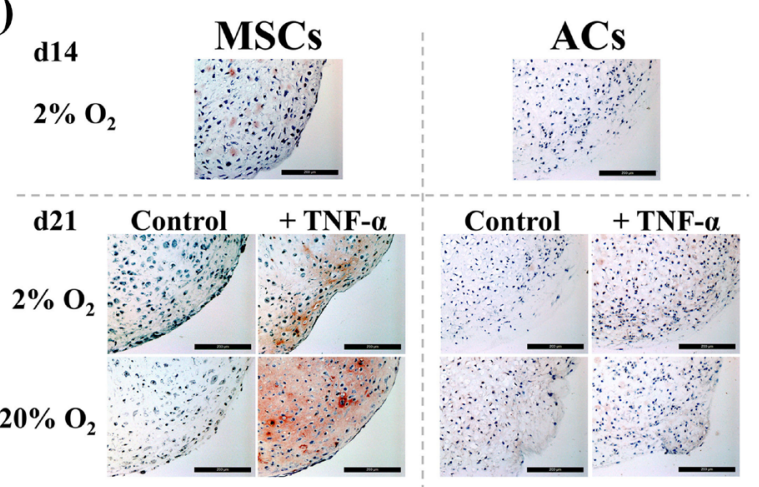

Fig. 3. Expression and activity of aggrecanases in MSC- and AC-derived neocartilage following TNF- $\alpha$ exposure and reoxygenation. Expression of ADAMTS4 (a) and ADAMTS5 (b) were analysed by qPCR using RNA from pellets of $n=4$ individual donors for each cell type at day $14\left(\mathrm{~d} 14: 2 \% \mathrm{O}_{2}\right.$ culture, pre-TNF- $\alpha$ exposure) and at day $21 \pm \mathrm{TNF}-\alpha$ with or without changes in oxygen. All values are the mean mRNA level normalised to $18 \mathrm{~S}$ ribosomal RNA and error bars represent one standard deviation. Statistical significance was determined by repeated measures ANOVA with Student-Newman-Keuls post hoc tests; $p<0.05$ vs. d14 (*), vs. matched oxygen level without TNF- $\alpha$ (\#), vs. low oxygen with TNF- $\alpha$ (\$). Catabolic activity mediated by aggrecanases was evaluated by (c) Western blotting for ARGS-bearing aggrecan fragments with BC-3 monoclonal antibody in supernatant collected and pooled from day 14 to day 21 and by (d) immunohistochemical staining for the NITEGE neoepitope in pellets at day 14 and day 21. Pellets of each experimental condition from one representative donor are shown. Scale bars $=200 \mu \mathrm{m}$. $\mathrm{L}=$ low oxygen $\left(2 \% \mathrm{O}_{2}\right), \mathrm{R}=$ reoxygenation $\left(20 \% \mathrm{O}_{2}\right)$.

cartilage, ADAMTS4 and ADAMTS5. Expression of the aggrecanases ADAMTS4 and ADAMTS5 in response to TNF- $\alpha$ treatment and oxygen tension was distinctly different in the MSCs and ACs (Fig. 3a,b). When MSCs were kept in hypoxia, TNF- $\alpha$ maintained ADAMTS4 expression at significantly higher levels than in untreated controls, but the combination of reoxygenation and TNF- $\alpha$ led to significantly higher levels than both their hypoxic-maintained counterparts and day 14 cells (Fig. 3a). For ACs, TNF- $\alpha$ increased ADAMTS4 expression to significantly higher levels than in untreated controls and day 14 expression. Additionally, concomitant reoxygenation did not affect the TNF- $\alpha$-induced changes in ADAMTS4 expression compared with hypoxic-maintained chondrocytes (Fig. 3a). In MSCs, TNF- $\alpha$ was a more potent stimulus of ADAMTS5 expression than ADAMTS4, with significant increases both compared to untreated controls and to day 14, regardless of oxygen levels during TNF- $\alpha$ treatment (Fig. 3b). Increases in ADAMTS5 were also significantly increased by reoxygenation compared with hypoxia. In contrast to MSCs, ADAMTS5 was not significantly affected by TNF- $\alpha$ nor by reoxygenation in ACs.

While ADAMTS4 and ADAMTS5 were elevated at the mRNA level, activity of these enzymes is only confirmed by evidence of generation of the aggrecan neoepitopes with termini ARGS and NITEGE. The involvement of aggrecanases in the TNF- $\alpha$-induced loss of proteoglycans from both MSC and AC pellets was assessed by immunoblotting for the aggrecan ARGS neoepitope in supernatants (Fig. 3c). In both MSC pellets and AC pellets maintained in medium without TNF- $\alpha$, ARGS-bearing aggrecan catabolites released into the medium were all low molecular mass with similar proportions of $\sim 64$ and $55 \mathrm{kDa}$ bands. In MSC pellets, TNF- $\alpha$ appeared to increase the release of high $(\sim 200 \mathrm{kDa})$, intermediate $(\sim 100 \mathrm{kDa})$, and low $(\sim 55 \mathrm{kDa})$ molecular mass ARGS-bearing aggrecan catabolites into the medium. Larger BC-3 positive fragments predominated in low oxygen and smaller fragments in re-oxygenation of MSC pellets treated with 
(a)

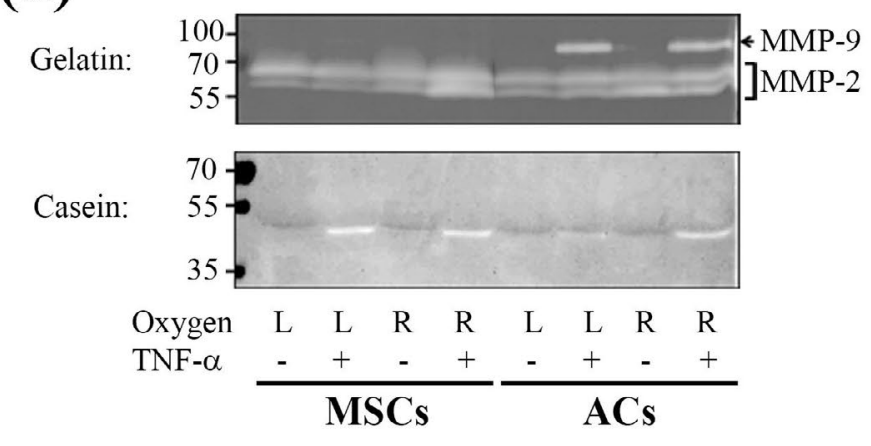

(b)

Fig. 4. Expression and activity of MMPs in MSCand AC-derived neocartilage following TNF- $\alpha$ exposure and reoxygenation. (a) Gelatinase and caseinase activity were evaluated by zymography using day 21 supernatants. MMP-9 presents as a single $92 \mathrm{kDa}$ band in the gelatine zymograms and MMP-2 presents as a doublet between 70 and $55 \mathrm{kDa}$, with the lower molecular weight band indicating active MMP-2. Gels from one representative donor of each cell type are shown. $\mathrm{L}=$ low oxygen $\left(2 \% \mathrm{O}_{2}\right), \mathrm{R}=$ reoxygenation $\left(20 \% \mathrm{O}_{2}\right)$. MMP activity was evaluated by immunohistochemical staining for (b) loss of collagen type II and (c) the appearance of the DIPEN aggrecan neoepitope in pellets at day 14 and day 21. Pellets of each experimental condition from one representative donor are shown. Scale bars $=200 \mu \mathrm{m}$.

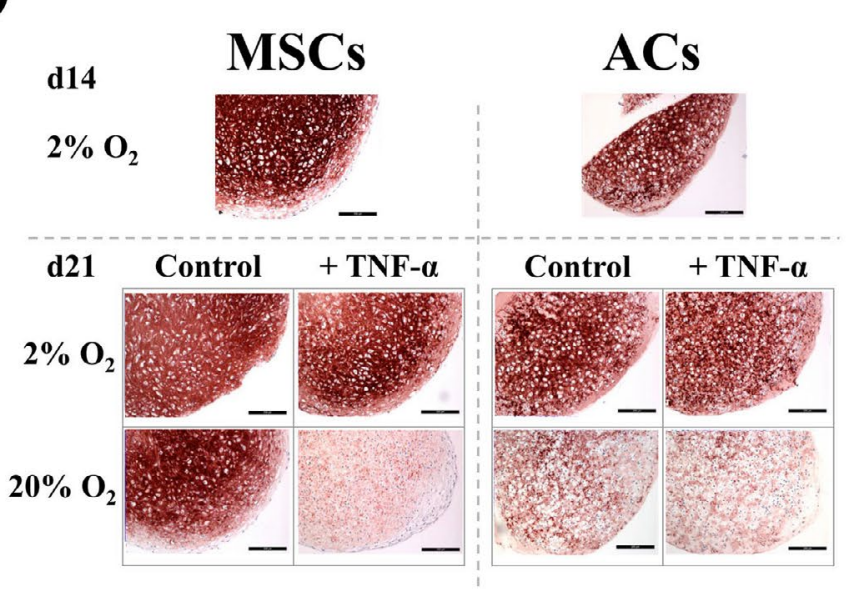

(c)

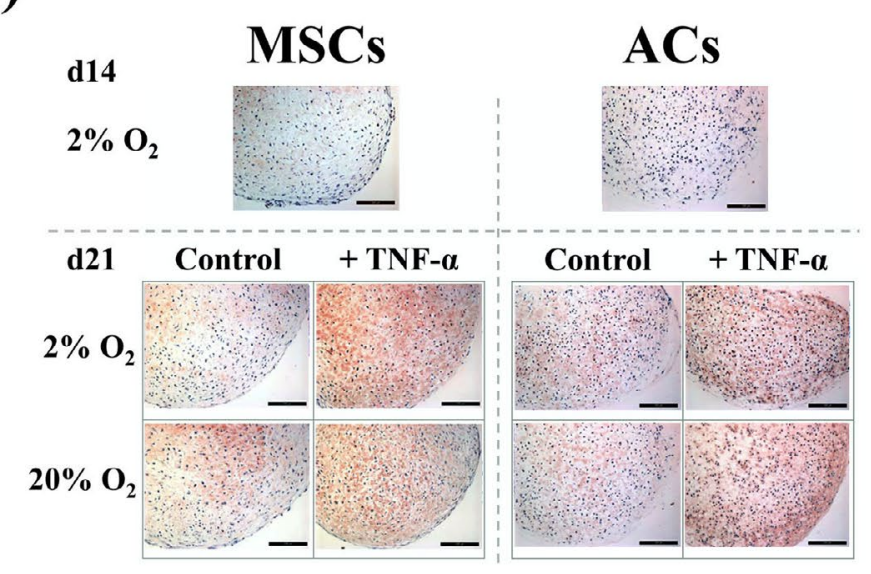

TNF- $\alpha$, evidenced by the apparently increased proportion of the $55 \mathrm{kDa}$ relative to the $64 \mathrm{kDa}$ fragment. In contrast, in AC pellets there was no release of large ARGS-bearing aggrecan catabolites into the medium in any cultures, although as in MSC pellets the combination of TNF- $\alpha$ and reoxygenation appeared to increase the proportion of $55 \mathrm{kDa}$ compared to $64 \mathrm{kDa}$ fragments. Immunostaining of the cell pellets for the NITEGE neoepitope confirmed ADAMTS-driven aggrecanolysis in MSCs treated with TNF- $\alpha$, particularly when combined with reoxygenation (Fig. 3d). In ACs pellets, there was only very faint NITEGE staining and no apparent difference between any condition (Fig. 3d), in agreement with the Western blots for ARGSbearing catabolites (Fig. 3c).

\section{Oxygen-dependent effects of TNF- $\alpha$ on MMP expression and activity}

Release of smaller molecular mass ARGS-bearing ADAMTS-cleaved aggrecan catabolites (Fig. 3c) has previously been associated with concomitant C-terminal proteolysis by MMPs (Jackson et al., 2014; Little et al., 2002). The presence of MMPs secreted into the medium was therefore evaluated by zymography. Gelatine zymography indicated that pro- and active forms of MMP-2 (middle and bottom bands, respectively) were secreted by both MSCs and ACs, and that in MSCs there was a consistently stronger lower band indicative of increased active MMP-2 when reoxygenation and TNF- $\alpha$ treatment were combined (Fig. 4a, top gel). Furthermore, 


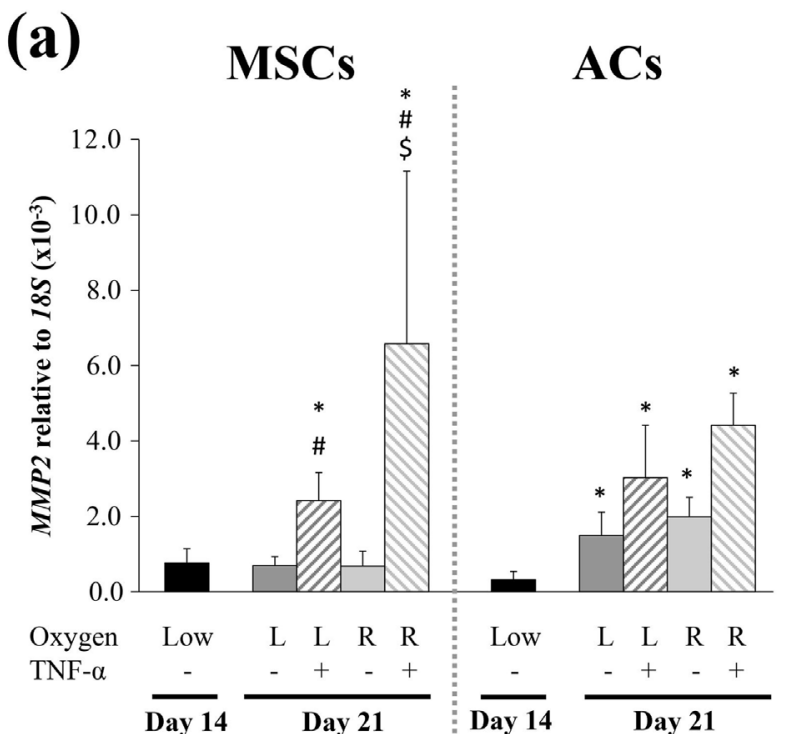

(c)

MSCs

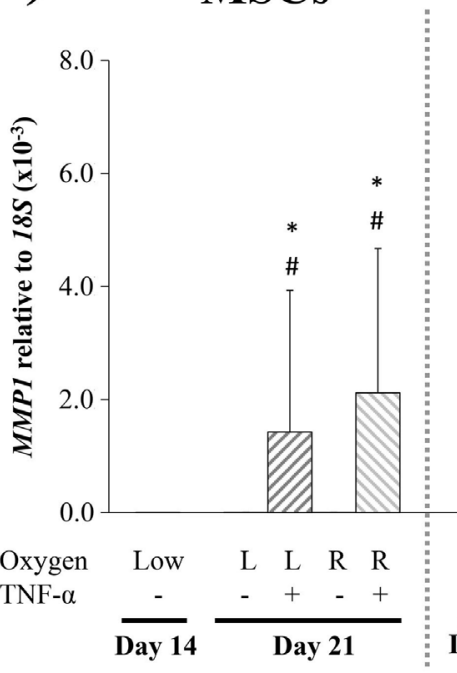

ACs

(e)

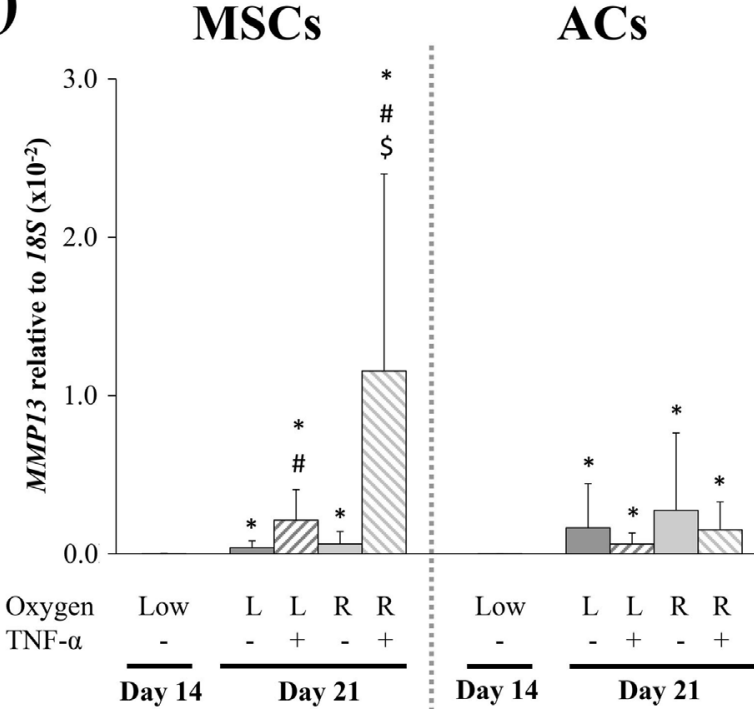

(b)

MSCs

ACs

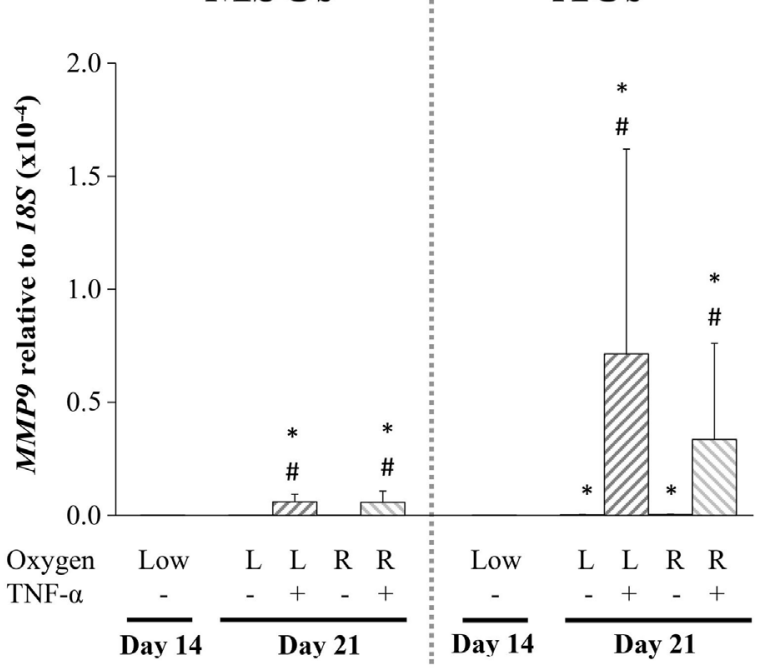

(d)

MSCs

ACs

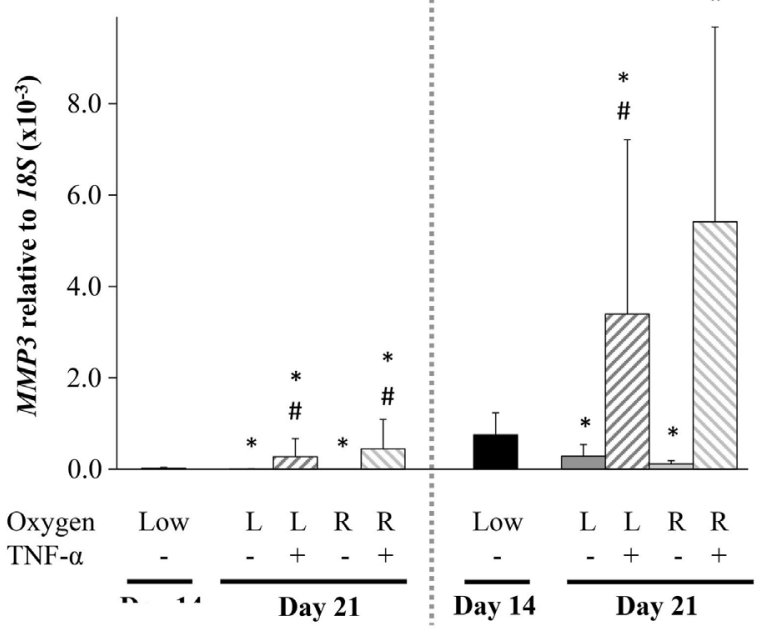

Fig. 5. Expression of mRNA for MMPs in MSC- and AC-derived neocartilage following TNF- $\alpha$ exposure and reoxygenation. Expression of $M M P 2$ (a), MMP9 (b), $M M P 1$ (c), MMP3 (d), and MMP13 (e) were analysed by qPCR using RNA from pellets of $n=4$ individual donors for each cell type at day $14\left(\mathrm{~d} 14: 2 \% \mathrm{O}_{2}\right.$ culture, preTNF- $\alpha$ exposure) and at day $21 \pm$ TNF- $\alpha$ with or without changes in oxygen. All values are the mean mRNA level normalised to $18 \mathrm{~S}$ ribosomal RNA and error bars represent one standard deviation. Statistical significance was determined by repeated measures ANOVA with Student-Newman-Keuls post hoc tests; $p<0.05$ vs. d14 $(*)$, vs. matched oxygen level without TNF- $\alpha(\#), v s$. low oxygen with TNF- $\alpha(\$)$. L = low oxygen $\left(2 \% \mathrm{O}_{2}\right)$, $\mathrm{R}=$ reoxygenation $\left(20 \% \mathrm{O}_{2}\right)$. 
when pellets were first cultured at $20 \%$ oxygen during chondrogenic induction, those MSC pellets kept at $20 \%$ oxygen during TNF- $\alpha$ exposure had consistently stronger bands indicative of active MMP-2 compared to those moved to $2 \%$ oxygen (data not shown). For ACs, we did not observe an effect of TNF- $\alpha$ on active MMP-2 generation, nor an effect of reoxygenation on TNF- $\alpha$ treated cells (Fig. 4a, top gel). In contrast, proMMP-9 (top band) accumulation in the medium was strongly induced by TNF- $\alpha$ in ACs independent of oxygen tension, whereas little to no proMMP-9 was detected in the supernatant from MSCs (Fig. 4a, top gel). Casein zymography revealed an EDTA-sensitive band at approximately $45 \mathrm{kDa}$ in supernatants from MSCs and ACs treated with TNF- $\alpha$ (Fig. 4a, bottom gel). This band was of similar to greater intensity in reoxygenated MSCs and ACs, depending on the sample. Candidate MMPs that could be responsible for this band include active forms of MMP-1, -3, -10, -12 and -13 (Snoek-van Beurden and von den Hoff, 2005).

Collagen II staining showed a similar pattern of matrix loss as that for aggrecan (Fig. 4b), indicating higher collagenolytic activity in reoxygenated TNF- $\alpha$-treated cells, particularly in MSCs. Consistent with this, and the increase in MMP activity in conditioned media of TNF$\alpha$-treated MSCs and ACs (Fig. 4a), there was staining indicative of an increase in the MMP-generated aggrecan DIPEN neoepitope in the pellets in these same cultures (Fig 4c). We were unable to demonstrate release of MMPgenerated C-terminal aggrecan fragments bearing the FFGV neoepitope in any cultures (data not shown).

Because there was evidence of differential MMP activity between cell types and conditions, we performed qPCR on several MMPs to determine which MMPs were upregulated in MSCs and ACs under these conditions. At the mRNA level, MMP2 expression was significantly increased by TNF- $\alpha$ compared with TNF- $\alpha$-free controls in MSCs in both oxygen conditions but not in ACs (Fig. 5a). Additionally, reoxygenation significantly increased TNF- $\alpha$-induced $M M P 2$ expression in MSCs. In agreement with the gelatine zymography, $M M P 9$ was not detectable in any of the untreated groups and was much lower in MSCs than in ACs following TNF- $\alpha$ exposure (Fig. 5b). Reoxygenation had no significant effect on TNF- $\alpha$-induced $M M P 9$ expression in either cell type.

TNF- $\alpha$ significantly increased $M M P 1$ expression in both oxygen levels for both MSCs and ACs and this was further increased by reoxygenation of ACs (Fig. 5c). In contrast to $M M P 1$, although $M M P 3$ expression was significantly higher in TNF- $\alpha$-treated MSCs and ACs than in their untreated controls (Fig. 5d), TNF- $\alpha$-induced $M M P 3$ expression was not affected by reoxygenation in either cell type. MMP13 expression was significantly increased by TNF- $\alpha$ compared with TNF- $\alpha$-free controls in MSCs in both oxygen conditions but not in ACs (Fig. 5e). Additionally, reoxygenation significantly increased TNF- $\alpha$-induced $M M P 13$ expression in MSCs. MMP12 was not consistently detectable in either MSCs or ACs even with TNF- $\alpha$ treatment, and MMP10 was highly expressed at day 14 in MSCs but not consistently detectable in any other condition for MSCs or any condition for ACs (data not shown).

\section{Discussion}

In this study, we evaluated the oxygen-dependent response to TNF- $\alpha$ in two types of neocartilage generated by three-dimensional (3D) cultures of healthy human ACs and chondrogenically differentiated human bone marrow-derived MSCs, respectively. Although we were unable to match the MSC and AC preparations by age, gender or pathology, our data indicate that maintaining a hypoxic environment mitigates TNF- $\alpha$-induced loss of proteoglycans from MSC-derived neocartilage as this tissue is more sensitive to increased oxygen during TNF- $\alpha$ exposure. However, AC-derived neocartilage is more sensitive to the withdrawal of chondrogenic factors, losing matrix and increasing expression of $M M P 1, M M P 2$, $M M P 9, M M P 13$ and $A D A M T S 5$ in response to the removal of TGF- $\beta 1$ and dexamethasone. This unforeseen difference is important to keep in mind when considering the differential loss in matrix observed between neocartilage made from MSCs compared to that made from ACs. While there was less loss of matrix from day 14 MSC-derived neocartilage kept under low oxygen conditions and treated with TNF- $\alpha$, the ability of MSCs to continue making matrix in the absence of chondrogenic factors likely at least partially accounts for their higher matrix retention.

As for an anti-inflammatory advantage of MSCs in terms of the response of the various enzymes measured, we found none: more of these genes were actually upregulated by TNF- $\alpha$ in MSCs than in ACs. However, it should be noted that MMP-9, which is considered a marker of latestage degeneration (Karsdal et al., 2008; Vincenti and Brinckerhoff, 2002), was dramatically induced at the protein level in ACs and not MSCs. The larger finding of the study was that the distinct expression profiles of degradative enzymes and aggrecan catabolites in TNF- $\alpha$ exposed AC and MSC-derived neocartilages indicate that the observed matrix degradation likely occurs through cell type-specific mechanisms. We have previously found that under long-term 3D culture expression of ADAMTS4 and ADAMTS5 as well as $M M P 1, M M P 2, M M P 3$ and $M M P 13$ in ACs were significantly decreased by hypoxic culture (Markway et al., 2013). Here we found that when similarly cultured ACs were challenged with an inflammatory stimulus, a hypoxic environment was insufficient to mitigate increases in any of these same genes except $M M P 1$. In contrast, maintaining a hypoxic environment significantly mitigated TNF- $\alpha$ induced expression of ADAMTS4, ADAMTS5, MMP 2 and MMP13 in neocartilage generated from MSCs.

Cytokine-induced expression of aggrecanase mRNA in cartilage varies by age and species (Caterson et al., 2000). In human ACs, we found that ADAMTS4 was more responsive to TNF- $\alpha$ induction than ADAMTS5, as previously reported (Song et al., 2007). In contrast, ADAMTS5 was significantly increased by TNF- $\alpha$ in MSCs compared with both day 14 and day 21 controls. ADAMTS4 expression in MSCs, however, was maintained at higher levels with TNF- $\alpha$ compared with non-treated controls but only increased from day 14 levels when TNF- $\alpha$ was combined with reoxygenation. While we have previously found both ADAMTS4 and ADAMTS5 to be 
decreased in healthy chondrocytes following long-term low oxygen culture (Markway et al., 2013), here we found no significant oxygen-dependent effect on TNF- $\alpha$-induced expression for either gene in ACs. This differed from MSCs that showed oxygen-dependent effects on TNF- $\alpha$-induced expression for both genes, with reoxygenation enhancing TNF- $\alpha$-induced aggrecanase expression.

Boeuf et al. (2012) reported that ADAMTS5 gene expression decreased and ADAMTS4 expression increased over the course of MSC chondrogenesis; however, aggrecan cleavage was not seen unless IL- $1 \beta$ was added, in which case mRNA levels of both ADAMTS4 and $A D A M T S 5$ increased and the aggrecanase-generated neoepitope, NITEGE, was detected. It was also noted in that study that the inducibility of ADAMTS 5 by IL- $1 \beta$ in MSCs differs from chondrocytes in that chondrocytes do not respond to IL-1 $\beta$ with an increase in ADAMTS5 expression (Boeuf et al., 2012). We found that TNF- $\alpha$ was also a potent inducer of ADAMTS5 in chondrogenically differentiated MSCs under either oxygen level investigated. Our results, combined with their observations, suggest that ADAMTS5 is not constitutively expressed in MSCderived chondrocytes, in contrast with chondrocytes, but is highly sensitive to inflammatory stimuli. However, it should be noted that the different expression patterns and apparently different sensitivities to TNF- $\alpha$ and oxygen in these cells is not necessarily indicative of divergent mechanisms for aggrecan loss. Although the combination of TNF- $\alpha$ and oncostatin M was previously shown to upregulate ADAMTS4 to a much greater degree than ADAMTS5 in both human chondrocytes and cartilage explants, siRNA targeting ADAMTS5 mitigated TNF- $\alpha$ / oncostatin M-induced loss of GAGs to a similar degree as that targeting ADAMTS4 (Song et al., 2007). Delineating differences in the relative contribution of these enzymes to aggrecan loss from neocartilage constructs of different origins will require more thorough investigation.

Analysis of aggrecanase-generated aggrecan fragments suggests that there is a mechanistic difference in the catabolic response of neocartilage derived from MSCs and ACs to oxygenation and TNF- $\alpha$. We found that in neocartilage derived from MSCs, TNF- $\alpha$ appeared to increase aggrecanolysis by ADAMTS, as previously shown with IL-1 $\beta$ (Boeuf et al., 2012) and that this is more pronounced with reoxygenation. We were unable to detect increased ADAMTS aggrecanolysis in AC-derived neocartilage, consistent with less upregulation of ADAMTS mRNA by TNF- $\alpha$ in ACs. There was also evidence of substantial aggrecan G1 loss from reoxygenated TNF- $\alpha$ stimulated pellets. Previous studies have shown that loss of aggrecan in response to specific cytokine stimulation may result not only from proteolysis but also depolymerisation of hyaluronan (Durigova et al., 2008; Sztrolovics et al., 2002a). Furthermore, reoxygenation can generate reactive oxygen species, which are well known to depolymerise hyaluronic acid (Bates et al., 1984; Mapp et al., 1995; McCord, 1974). Our data showing the most profound loss of G1 with TNF- $\alpha$ in combination with reoxygenation, suggests a role for reactive oxygen species in hyaluronan degradation and proteoglycan loss here.
MMPs can also cleave aggrecan; however, in native cartilage these enzymes are thought to contribute little directly to inflammatory factor-mediated aggrecan loss, at least in the short-term (Durigova et al., 2011; Little et al., 1999; Madsen et al., 2010; Struglics and Hansson, 2012). There are six known MMP cleavage sites in human aggrecan (Struglics and Hansson, 2012) with the major site between $\mathrm{Asn}^{341}$ and $\mathrm{Phe}^{342}$ resulting in fragments terminating with the sequences DIPEN ${ }^{341}$ (DIPES $^{341}$ in bovine) and ${ }^{342} \mathrm{FFGVG}$. Little et al. (1999) found that cleavage of aggrecan at this site inconsequentially contributed to TNF- $\alpha$-induced loss of proteoglycans. Similarly, Fosang et al. (2000) estimated that MMPs account for less than $1 \%$ of aggrecan released upon catabolic stimulation. However, the contribution of MMPs to aggrecan degradation in neocartilage has not been extensively studied. Aggrecanolysis by MMPs varies by species and tissue type as well as by catabolic stimuli (Fuller et al., 2012; Hughes et al., 1998; Little et al., 1999; Sztrolovics et al., 2002b). Little et al. (1999) reported an absence of ${ }^{342} \mathrm{FFGVG}$ fragments in culture supernatants from bovine, porcine and human articular cartilage explants treated for four days with catabolic stimuli and little evidence of increased DIPEN/S accumulation in the matrix. Subsequently, they reported that in the third week of IL-1 treatment, both FFGVG fragments and DIPEN/S accumulated in the medium of bovine nasal cartilage (Little et al., 2002). In ovine outer meniscus explant cultures TNF- $\alpha$ increased the generation of DIPEN fragments but not NITEGE (Fuller et al., 2012). This is of interest because the inner meniscus responded similarly to articular cartilage with an increase in NITEGE but not DIPEN fragments. Thus, the differences in the phenotype and matrix created by MSC-derived chondrocytes compared with articular chondrocytes may also include different matrix-degrading proenzymes leading to a distinct cascade of degradative events. However, Boeuf et al. (2012) found that the response of MSC-derived neocartilage to IL-1 $\beta$ was similar to that of articular chondrocytes: a dramatic increase in NITEGE fragments with little effect on generation of DIPEN. Apart from this study, we are not aware of any published studies investigating the inflammatory-induced aggrecanolytic response of neocartilage, and none that examine the effects of oxygen tension in aggrecanolysis. Therefore, in addition to analysis of the major aggrecanases, we evaluated the generation of MMP-derived neoepitopes and the expression of MMPs that could be involved in this process.

Unlike previous studies with IL-1 $\beta$ (Boeuf et al., 2012) our immunohistochemical data also demonstrated increased aggrecanolysis by MMPs in response to TNF- $\alpha$ in both MSC- and AC-derived neocartilage. However, that our data suggests increased DIPEN generation but not an accompanying release of FFGV-bearing C-terminal aggrecan catabolites in the culture medium suggests that MMP-mediated cleavage may be occurring secondary to primary interglobular domain proteolysis by ADAMTS, with the resultant 32-amino acid peptide being lost during dialysis of samples prior to Western blotting. The reduced size of ARGS-bearing aggrecan fragments with TNF- $\alpha$ in 
the presence of reoxygenation may indicate C-terminal proteolysis of aggrecan by MMPs (Jackson et al., 2014; Little et al., 2002), and was consistent with greater type II collagen loss in these same pellets.

In ACs, there was no significant effect on $M M P 2$ expression and no noticeable effect on generation of active MMP-2 upon TNF- $\alpha$ exposure, a result in agreement with previous studies of articular cartilage and chondrocytes (Fuller et al., 2012; Lefebvre et al., 1991). Additionally, oxygen did not modulate the effects of TNF- $\alpha$ on MMP2 . In contrast, $M M P 2$ was increased by TNF- $\alpha$ in MSCs and this was enhanced by reoxygenation. TNF- $\alpha$-induced generation of active MMP-2 also appeared enhanced by reoxygenation of these cells. Since TNF- $\alpha$ had little effect on the generation of active MMP-2 in articular cartilage and inner meniscus but did increase levels of active MMP2 in outer meniscus (Fuller et al., 2012), the differences we find may reflect a more fibrocartilage phenotype of MSC-derived chondrocytes: akin to outer meniscal cells with higher COL1A1 and lower COL2A1 expression (Fuller et al., 2012; Ochi et al., 2003). It is relevant that MMP-2 has been shown to be activated by collagen I in several experimental systems (Guo and Piacentini, 2003; Henderson et al., 2007; Wang et al., 2003) and its abundance in MSC-derived neocartilage may potentiate the TNF- $\alpha$ induced activation of MMP-2. The lower level of active MMP-2 induced by TNF- $\alpha$ in MSCs maintained in hypoxia corresponds with the significantly lower COL1A1 in the day 14 MSC pellets differentiated in hypoxia compared with normoxia (data not shown). The role of collagen I in inflammatory factor-mediated activation of MMP-2 deserves more thorough investigation.

The response of the other gelatinase, MMP-9, was also distinctly different between MSC- and AC-derived neocartilage. While MMP9 mRNA was only ever detectable following TNF- $\alpha$ treatment for both cell types, in MSCs it rarely increased to a level such that the protein could be detected by zymography. In contrast, in supernatant from ACs, MMP-9 was always present in detectable amounts and this was reflected in the different levels of $M M P 9$ between the two cell types. Very little to no MMP-9 was reported in the supernatant from any meniscal or cartilage explants (Fuller et al., 2012). The fact that we found substantial MMP-9 in the supernatants from articular chondrocytes may be due to species differences and/or differences in the culture system, i.e. explants of native cartilage compared with neocartilage from redifferentiated chondrocytes. MSCs have a low level of $M M P 9$ expression even following chondrogenesis and respond to TNF- $\alpha$ with less elevated levels of $M M P 9$ than ACs. Since MMP-9 is a gelatinase that only cleaves collagen after the chains of the triple helix have been cleaved by collagenases (such as MMP-1), it may be more involved in late-stage degeneration (Vincenti and Brinckerhoff, 2002). Karsdal et al. (2008) noted that in bovine cartilage explants elevated levels of MMP-2 and MMP-9 in the medium corresponded with a late stage of degradation marked as a point of severely impaired capacity for reversing the damage. Our results show most dramatically that after one week of TNF- $\alpha$, proMMP-9 is substantially elevated in AC pellets compared to MSC pellets. This may indicate that the AC-derived neocartilage reaches the severe stage of degeneration quicker than MSCderived neocartilage under these inflammatory conditions. Future studies should investigate this potential advantage of MSCs by examining the degradation of collagen and the reversibility of damage in MSC-derived neocartilage compared to AC-based neocartilage.

MMP-1 and MMP-3 are increased by TNF- $\alpha$ at both the mRNA and protein level in cultured chondrocytes (Fuller et al., 2012; Tetlow et al., 2001). Here we found that MMP-3, considered to be the major aggrecandegrading MMP of cartilage (Durigova et al., 2011), was significantly increased by TNF- $\alpha$ in AC-derived neocartilage with no modulation by oxygen. While $M M P 3$ expression was induced by TNF- $\alpha$ in MSCs, it remained at much lower levels than in ACs both with and without TNF- $\alpha$. As with $M M P 9$, induction of $M M P 3$ appears to be a minor part of the TNF- $\alpha$ response of MSC-derived neocartilage in comparison to AC-derived neocartilage. $M M P 1$, on the other hand, was expressed at a similar level in ACs and MSCs following TNF- $\alpha$ stimulation, although reoxygenation only significantly enhanced the effect in ACs.

Only MSCs demonstrate a significant upregulation of $M M P 13$ expression in response to TNF- $\alpha$ exposure, and this gene was further upregulated in response to reoxygenation. MMP13 is a marker of the hypertrophic phenotype as the MMP-13 enzyme preferentially degrades type II collagen to facilitate endochondral ossification. MSCs progress toward a hypertrophic phenotype in chondrogenic differentiation as compared with ACs (Fischer et al., 2010; Markway et al., 2015). In addition to its physiologic role in chondrocyte hypertrophy, MMP-13 is implicated in the pathogenesis of inflammatory arthritis through the recruitment of inflammatory cells and cytokines to articular cartilage (Joronen et al., 2004; Singh et al., 2013), and the enzyme may participate in positive feedback by both stimulating and responding to inflammation, including TNF- $\alpha$ exposure. As noted for MMP-2 above, TNF- $\alpha$ was previously reported to have no effect on MMP13 at the gene or protein level in articular cartilage and inner meniscus but increased levels of MMP13 in lateral outer meniscus (Fuller et al., 2012; Tetlow et al., 2001); again the differences we find may reflect a more fibrocartilage phenotype of MSC-derived chondrocytes.

We have not yet elucidated mechanisms for the observed protective effect of hypoxia and the additive effect of reoxygenation on TNF- $\alpha$-mediated damage. However, studies in which cells were cultured for two weeks in high oxygen and then moved to hypoxia (data not shown) revealed largely reciprocal and confirmatory results to the reoxygenation results presented here. That the effects were similarly inverse across several genes when cells were reoxygenated compared with when they were introduced to hypoxia is interesting with respect to hypoxic feedback mechanisms and our previous observations on the dynamics of HIF expression during long-term culture. It has been shown that hypoxia leads to increased prolyl hydroxylase expression and this can act to rapidly degrade HIFs upon reoxygenation (D'Angelo et al., 2003). We have previously seen that over two weeks of culture 
in hypoxia, both HIF-1 $\alpha$ and HIF- $2 \alpha$ protein levels in chondrocytes decrease dramatically over time (Markway et al., 2013) and we have observed that MSCs exhibit a similar decrease in HIF- $1 \alpha$ and HIF-2 $\alpha$ expression during long-term hypoxic chondrogenesis (unpublished results). Therefore, while reoxygenation can have dramatic effects on tissue integrity as shown here and by Strobel et al. (2010), changes in HIF- $1 \alpha$ and HIF- $2 \alpha$ protein levels would be expected to be small compared with the opposite condition of a first-time hypoxic exposure. It may be that there is a low level of stabilised HIF proteins following chronic hypoxia of chondrogenic cells but a level that is critical to the protective effects of hypoxia during TNF- $\alpha$ stimulation. How exactly hypoxic signalling counteracts the effects of TNF- $\alpha$ and the roles of the individual HIFs remains to be determined.

Modulation of nitric oxide (NO) synthesis is another intriguing possibility to explain our results. NO production is often elevated in the diseased joint and it is suggested to be an important catabolic factor contributing to OA progression (Abramson, 2008). In bovine cartilage explants cultures, a nitric oxide synthase (NOS) inhibitor was found to significantly attenuate the TNF- $\alpha$-mediated loss of sGAGs and to decrease generation of the NITEGE fragment but did not affect expression of ADAMTS4 or ADAMTS5 (Stevens et al., 2008). This suggested that NO contributes to TNF- $\alpha$-mediated aggrecanolysis at a post-transcriptional level. Hypoxia was found to decrease TNF- $\alpha$-induced NO production in porcine explant cultures but reoxygenation enhanced TNF- $\alpha$-induced NO production (Cernanec et al., 2002). Hypoxia/reoxygenation events have also been shown to increase TNF- $\alpha$-induced expression of inducible NOS (iNOS) in synoviocytes from OA patients (Chenevier-Gobeaux et al., 2013). It has been shown that following chondrogenic differentiation, in contrast to undifferentiated and osteogenic and adipogenic cultures, MSCs express iNOS and produce NO upon IL$1 \beta$ stimulation (Mais et al., 2006). In that study, MSCs produced lower levels of NO than chondrocytes following IL- $1 \beta$ stimulation, but these were not in comparable culture systems (alginate $v s$. pellets) and the chondrocytes were derived from diseased joints. It would be interesting to compare the NO production of chondrogenically induced MSCs to that of healthy chondrocytes in parallel culture systems and to evaluate how oxygen tension modulates inflammatory-mediated NO synthesis in these two cell types.

\section{Conclusions}

Relatively little is known about how the hypoxic environment and disease-associated changes in oxygen tension affect joint degeneration. The majority of in vitro hypoxia studies focus on the anabolic effects, often with an eye towards increasing matrix production for tissue engineering applications. Our results provide further evidence of the need to consider oxygen an essential parameter for studying the degradative axis. Specifically, we show that oxygen can modulate cytokine-mediated expression of degradative enzymes and that this effect differs depending on the cell type. Furthermore, despite the purported anti-inflammatory properties of naïve MSCs, we found no definitive advantage of MSC-derived neocartilage as it relates to expression of the enzymes typically associated with loss of cartilage matrix. These results have important implications when considering implantation into diseased joint environments and for the selection of appropriate biological agents, such as enzyme inhibitors, to protect the new tissue and augment repair.

\section{Acknowledgments}

The authors thank the following surgeons in OHSU Orthopaedics: Dr. Dennis Crawford for providing human healthy cartilage samples and Drs. Jung Yoo, Alex Ching and Bob Hart for providing bone marrow. We also acknowledge the immunohistochemistry skills of Ms. Susan Smith. The research was supported in part by grants from the Collins Medical Trust and the Arthritis Foundation (BDM) and the Australian National Health and Medical Research Council (NHMRC) project grants APP1045890 and APP1063133 (CBL). Dr. Johnstone patented the technology for the in vitro method for differentiating mesenchymal stem cells into chondrocytes and it is licensed to Osiris Therapeutics, Inc. We wish to confirm that there are no known conflicts of interest associated with this publication and there has been no significant financial support for this work that could have influenced its outcome.

\section{References}

Abramson SB (2008) Nitric oxide in inflammation and pain associated with osteoarthritis. Arthritis research \& therapy 10 Suppl 2: S2.

Bates EJ, Harper GS, Lowther DA, Preston BN (1984) Effect of oxygen-derived reactive species on cartilage proteoglycan-hyaluronate aggregates. Biochem Int 8: 629637.

Blake DR, Merry P, Unsworth J, Kidd BL, Outhwaite JM, Ballard R, Morris CJ, Gray L, Lunec J (1989) Hypoxicreperfusion injury in the inflamed human joint. Lancet 1: 289-293.

Boeuf S, GrafF, Fischer J, Moradi B, Little CB, Richter W (2012) Regulation of aggrecanases from the ADAMTS family and aggrecan neoepitope formation during in vitro chondrogenesis of human mesenchymal stem cells. Eur Cell Mater 23: 320-332.

Bohensky J, Terkhorn SP, Freeman TA, Adams CS, Garcia JA, Shapiro IM, Srinivas V (2009) Regulation of autophagy in human and murine cartilage: hypoxiainducible factor 2 suppresses chondrocyte autophagy. Arthritis Rheum 60: 1406-1415.

Brighton CT, Heppenstall RB (1971) Oxygen tension of the epiphyseal plate distal to an arteriovenous fistula. Clin Orthop Relat Res 80: 167-173.

Buttle DJ, Fowles A, Ilic MZ, Handley CJ (1997) "Aggrecanase" activity is implicated in tumour necrosis 
factor alpha mediated cartilage aggrecan breakdown but is not detected by an in vitro assay. Mol Pathol 50: 153-159.

Caterson B, Flannery CR, Hughes CE, Little CB (2000) Mechanisms involved in cartilage proteoglycan catabolism. Matrix Biol 19: 333-344.

Cernanec J, Guilak F, Weinberg JB, Pisetsky DS, Fermor B (2002) Influence of hypoxia and reoxygenation on cytokine-induced production of proinflammatory mediators in articular cartilage. Arthritis Rheum 46: 968975.

Chenevier-Gobeaux C, Simonneau C, Lemarechal H, Bonnefont-Rousselot D, Poiraudeau S, Rannou F, Ekindjian OG, Anract P, Borderie D (2013) Effect of hypoxia/reoxygenation on the cytokine-induced production of nitric oxide and superoxide anion in cultured osteoarthritic synoviocytes. Osteoarthritis Cartilage 21: 874-881.

Clerigues V, Murphy CL, Guillen MI, Alcaraz MJ (2013) Haem oxygenase-1 induction reverses the actions of interleukin-1beta on hypoxia-inducible transcription factors and human chondrocyte metabolism in hypoxia. Clinical Sci 125: 99-108.

Coimbra IB, Jimenez SA, Hawkins DF, PieraVelazquez S, Stokes DG (2004) Hypoxia inducible factor-1 alpha expression in human normal and osteoarthritic chondrocytes. Osteoarthritis Cartilage 12: 336-345.

D’Angelo G, Duplan E, Boyer N, Vigne P, Frelin C (2003) Hypoxia up-regulates prolyl hydroxylase activity: a feedback mechanism that limits HIF-1 responses during reoxygenation. J Biol Chem 278: 38183-38187.

Durigova M, Roughley PJ, Mort JS (2008) Mechanism of proteoglycan aggregate degradation in cartilage stimulated with oncostatin M. Osteoarthritis Cartilage 16: 98-104.

Durigova M, Nagase H, Mort JS, Roughley PJ (2011) MMPs are less efficient than ADAMTS5 in cleaving aggrecan core protein. Matrix Biol 30: 145-153.

Fischer J, Dickhut A, Rickert M, Richter W (2010) Human articular chondrocytes secrete parathyroid hormonerelated protein and inhibit hypertrophy of mesenchymal stem cells in coculture during chondrogenesis. Arthritis Rheum 62: 2696-2706.

Flannery CR, Little CB, Hughes CE, Curtis CL, Caterson B, Jones SA (2000) IL-6 and its soluble receptor augment aggrecanase-mediated proteoglycan catabolism in articular cartilage. Matrix Biol 19: 549-553.

Fosang AJ, Last K, Stanton H, Weeks DB, Campbell IK, Hardingham TE, Hembry RM (2000) Generation and novel distribution of matrix metalloproteinase-derived aggrecan fragments in porcine cartilage explants. J Biol Chem 275: 33027-33037.

Fuller ES, Smith MM, Little CB, Melrose J (2012) Zonal differences in meniscus matrix turnover and cytokine response. Osteoarthritis Cartilage 20: 49-59.

Giatromanolaki A, Sivridis E, Maltezos E, Athanassou N, Papazoglou D, Gatter KC, Harris AL, Koukourakis MI (2003) Upregulated hypoxia inducible factor-1alpha and -2alpha pathway in rheumatoid arthritis and osteoarthritis. Arthritis Res Ther 5: R193-201.

Guo C, Piacentini L (2003) Type I collagen-induced MMP-2 activation coincides with up-regulation of membrane type 1-matrix metalloproteinase and TIMP-2 in cardiac fibroblasts. J Biol Chem 278: 46699-46708.

Hashimoto K, Otero M, Imagawa K, de Andres MC, Coico JM, Roach HI, Oreffo RO, Marcu KB, Goldring MB (2013) Regulated transcription of human matrix metalloproteinase 13 (MMP13) and interleukin-1beta (IL1B) genes in chondrocytes depends on methylation of specific proximal promoter $\mathrm{CpG}$ sites. J Biol Chem 288: 10061-10072.

Henderson N, Markwick LJ, Elshaw SR, Freyer AM, Knox AJ, Johnson SR (2007) Collagen I and thrombin activate MMP-2 by MMP-14-dependent and -independent pathways: implications for airway smooth muscle migration. Am J Physiol Lung Cell Mol Physiol 292: L1030-1038.

Higuchi H, Shirakura K, Kimura M, Terauchi M, Shinozaki T, Watanabe H, Takagishi K (2006) Changes in biochemical parameters after anterior cruciate ligament injury. Int Orthop 30: 43-47.

Hollander AP, Corke KP, Freemont AJ, Lewis CE (2001) Expression of hypoxia-inducible factor 1alpha by macrophages in the rheumatoid synovium: implications for targeting of therapeutic genes to the inflamed joint. Arthritis Rheum 44: 1540-1544.

Hughes CE, Little CB, Buttner FH, Bartnik E, Caterson B (1998) Differential expression of aggrecanase and matrix metalloproteinase activity in chondrocytes isolated from bovine and porcine articular cartilage. J Biol Chem 273: 30576-30582.

Jackson MT, Moradi B, Smith MM, Jackson CJ, Little CB (2014) Activation of matrix metalloproteinases 2, 9, and 13 by activated protein $\mathrm{C}$ in human osteoarthritic cartilage chondrocytes. Arthritis Rheum 66: 1525-1536.

Johnstone B, Hering TM, Caplan AI, Goldberg VM, Yoo JU (1998) In vitro chondrogenesis of bone marrowderived mesenchymal progenitor cells. Exp Cell Res 238: 265-272.

Joronen K, Ala-aho R, Majuri ML, Alenius H, Kahari VM, Vuorio E (2004) Adenovirus mediated intraarticular expression of collagenase-3 (MMP-13) induces inflammatory arthritis in mice. Ann Rheum Dis 63: 656664.

Kanichai M, Ferguson D, Prendergast PJ, Campbell VA (2008) Hypoxia promotes chondrogenesis in rat mesenchymal stem cells: a role for AKT and hypoxiainducible factor (HIF)-1alpha. J Cell Physiol 216: 708-715.

Karsdal MA, Madsen SH, Christiansen C, Henriksen K, Fosang AJ, Sondergaard BC (2008) Cartilage degradation is fully reversible in the presence of aggrecanase but not matrix metalloproteinase activity. Arthritis Res Ther 10: R63.

Lafont JE, Talma S, Murphy CL (2007) Hypoxiainducible factor 2alpha is essential for hypoxic induction of the human articular chondrocyte phenotype. Arthritis Rheum 56: 3297-3306.

Lafont JE, Talma S, Hopfgarten C, Murphy CL (2008) Hypoxia promotes the differentiated human articular chondrocyte phenotype through SOX9-dependent and -independent pathways. J Biol Chem 283: 4778-4786.

Larsson S, Englund M, Struglics A, Lohmander LS (2015) Interleukin-6 and tumor necrosis factor alpha 
in synovial fluid are associated with progression of radiographic knee osteoarthritis in subjects with previous meniscectomy. Osteoarthritis Cartilage 23: 1906-1914.

Lefebvre V, Peeters-Joris C, Vaes G (1991) Production of gelatin-degrading matrix metalloproteinases ('type IV collagenases') and inhibitors by articular chondrocytes during their dedifferentiation by serial subcultures and under stimulation by interleukin-1 and tumor necrosis factor alpha. Biochimt Biophys Acta 1094: 8-18.

Little CB, Flannery CR, Hughes CE, Mort JS, Roughley PJ, Dent C, Caterson B (1999) Aggrecanase versus matrix metalloproteinases in the catabolism of the interglobular domain of aggrecan in vitro. Biochem J 344: 61-68.

Little CB, Hughes CE, Curtis CL, Janusz MJ, Bohne R, Wang-Weigand S, Taiwo YO, Mitchell PG, Otterness IG, Flannery CR, Caterson B (2002) Matrix metalloproteinases are involved in $\mathrm{C}$-terminal and interglobular domain processing of cartilage aggrecan in late stage cartilage degradation. Matrix Biol 21: 271-288.

Lund-Olesen K (1970) Oxygen tension in synovial fluids. Arthritis Rheum 13: 769-776.

Madsen SH, Sumer EU, Bay-Jensen AC, Sondergaard BC, Qvist P, Karsdal MA (2010) Aggrecanase- and matrix metalloproteinase-mediated aggrecan degradation is associated with different molecular characteristics of aggrecan and separated in time ex vivo. Biomarkers 15: 266-276.

Mais A, Klein T, Ullrich V, Schudt C, Lauer G (2006) Prostanoid pattern and iNOS expression during chondrogenic differentiation of human mesenchymal stem cells. J Cell Biochem 98: 798-809.

Mapp PI, Grootveld MC, Blake DR (1995) Hypoxia, oxidative stress and rheumatoid arthritis. Br Med Bull 51: 419-436.

Markway BD, Tan GK, Brooke G, Hudson JE, CooperWhite JJ, Doran MR (2010) Enhanced chondrogenic differentiation of human bone marrow-derived mesenchymal stem cells in low oxygen environment micropellet cultures. Cell Transplant 19: 29-42.

Markway BD, Cho H, Johnstone B (2013) Hypoxia promotes redifferentiation and suppresses markers of hypertrophy and degeneration in both healthy and osteoarthritic chondrocytes. Arthritis Res Ther 15: R92.

Markway BD, Cho H, Zilberman-Rudenko J, Holden P, McAlinden A, Johnstone B (2015) Hypoxia-inducible factor 3-alpha expression is associated with the stable chondrocyte phenotype. J Orthop Res 33: 1561-1570.

Martin G, Andriamanalijaona R, Grassel S, Dreier R, Mathy-Hartert M, Bogdanowicz P, Boumediene K, Henrotin Y, Bruckner P, Pujol JP (2004) Effect of hypoxia and reoxygenation on gene expression and response to interleukin-1 in cultured articular chondrocytes. Arthritis Rheum 50: 3549-3560.

McCord JM (1974) Free radicals and inflammation: protection of synovial fluid by superoxide dismutase. Science 185: 529-531.

Ochi K, Daigo Y, Katagiri T, Saito-Hisaminato A, Tsunoda T, Toyama Y, Matsumoto H, Nakamura Y (2003) Expression profiles of two types of human knee-joint cartilage. J Hum Genet 48: 177-182.
Prockop DJ, Oh JY (2012) Mesenchymal stem/stromal cells (MSCs): role as guardians of inflammation. Mol Ther 20: 14-20.

Robins JC, Akeno N, Mukherjee A, Dalal RR, Aronow BJ, Koopman P, Clemens TL (2005) Hypoxia induces chondrocyte-specific gene expression in mesenchymal cells in association with transcriptional activation of Sox 9. Bone 37: 313-322.

Saito T, Fukai A, Mabuchi A, Ikeda T, Yano F, Ohba S, Nishida N, Akune T, Yoshimura N, Nakagawa T, Nakamura K, Tokunaga K, Chung UI, Kawaguchi H (2010) Transcriptional regulation of endochondral ossification by HIF-2alpha during skeletal growth and osteoarthritis development. Nat Med 16: 678-686.

Silver IA (1975) Measurement of $\mathrm{pH}$ and ionic composition of pericellular sites. Philos Trans R Soc Lond B Biol Sci 271: 261-272.

Singh A, Rajasekaran N, Hartenstein B, Szabowski S, Gajda M, Angel P, Brauer R, Illges H (2013) Collagenase-3 (MMP-13) deficiency protects C57BL/6 mice from antibody-induced arthritis. Arthritis Res Ther 15: R222.

Snoek-van Beurden PA, von den Hoff JW (2005) Zymographic techniques for the analysis of matrix metalloproteinases and their inhibitors. Biotechniques 38: 73-83.

Song RH, Tortorella MD, Malfait AM, Alston JT, Yang Z, Arner EC, Griggs DW (2007) Aggrecan degradation in human articular cartilage explants is mediated by both ADAMTS-4 and ADAMTS-5. Arthritis Rheum 56: 575585.

Stevens AL, Wheeler CA, Tannenbaum SR, Grodzinsky AJ (2008) Nitric oxide enhances aggrecan degradation by aggrecanase in response to TNF-alpha but not IL-1beta treatment at a post-transcriptional level in bovine cartilage explants. Osteoarthritis Cartilage 16: 489-497.

Strobel S, Loparic M, Wendt D, Schenk AD, Candrian C, Lindberg RL, Moldovan F, Barbero A, Martin I (2010) Anabolic and catabolic responses of human articular chondrocytes to varying oxygen percentages. Arthritis Res Ther 12: R34.

Struglics A, Hansson M (2012) MMP proteolysis of the human extracellular matrix protein aggrecan is mainly a process of normal turnover. Biochem J 446: 213-223.

Sztrolovics R, Recklies AD, Roughley PJ, Mort JS (2002a) Hyaluronate degradation as an alternative mechanism for proteoglycan release from cartilage during interleukin-1beta-stimulated catabolism. Biochem J 362: 473-479.

Sztrolovics R, White RJ, Roughley PJ, Mort JS (2002b) The mechanism of aggrecan release from cartilage differs with tissue origin and the agent used to stimulate catabolism. Biochem J 362: 465-472.

Tetlow LC, Adlam DJ, Woolley DE (2001) Matrix metalloproteinase and proinflammatory cytokine production by chondrocytes of human osteoarthritic cartilage: associations with degenerative changes. Arthritis Rheum 44: 585-594.

Thoms BL, Dudek KA, Lafont JE, Murphy CL (2013) Hypoxia promotes the production and inhibits the destruction of human articular cartilage. Arthritis Rheum 65: $1302-1312$. 
Tsuchida AI, Beekhuizen M, Rutgers M, van Osch GJ, Bekkers JE, Bot AG, Geurts B, Dhert WJ, Saris DB, Creemers LB (2012) Interleukin-6 is elevated in synovial fluid of patients with focal cartilage defects and stimulates cartilage matrix production in an in vitro regeneration model. Arthritis Res Ther 14: R262.

Tsuchida AI, Beekhuizen M, t Hart MC, Radstake TR, Dhert WJ, Saris DB, van Osch GJ, Creemers LB (2014) Cytokine profiles in the joint depend on pathology, but are different between synovial fluid, cartilage tissue and cultured chondrocytes. Arthritis Res Ther 16: 441.

Vincenti MP, Brinckerhoff CE (2002) Transcriptional regulation of collagenase (MMP-1, MMP-13) genes in arthritis: integration of complex signaling pathways for the recruitment of gene-specific transcription factors. Arthritis Res 4: 157-164.

Vos LM, Slater JJ, Leijsma MK, Stegenga B (2012) Does hypoxia-reperfusion injury occur in osteoarthritis of the temporomandibular joint? J Orofac Pain 26: 233-239.

Wang DR, Sato M, Li LN, Miura M, Kojima N, Senoo H (2003) Stimulation of pro-MMP-2 production and activation by native form of extracellular type I collagen in cultured hepatic stellate cells. Cell Struct Funct 28: 505-513.

Wehling N, Palmer GD, Pilapil C, Liu F, Wells JW, Muller PE, Evans CH, Porter RM (2009) Interleukin-1beta and tumor necrosis factor alpha inhibit chondrogenesis by human mesenchymal stem cells through NF-kappaBdependent pathways. Arthritis Rheum 60: 801-812.

Westacott CI, Whicher JT, Barnes IC, Thompson D, Swan AJ, Dieppe PA (1990) Synovial fluid concentration of five different cytokines in rheumatic diseases. Ann Rheum Dis 49: 676-681.

Westacott CI, Barakat AF, Wood L, Perry MJ, Neison P, Bisbinas I, Armstrong L, Millar AB, Elson CJ (2000) Tumor necrosis factor alpha can contribute to focal loss of cartilage in osteoarthritis. Osteoarthritis Cartilage 8: 213-221.

Yang S, Kim J, Ryu JH, Oh H, Chun CH, Kim BJ, Min $\mathrm{BH}$, Chun JS (2010) Hypoxia-inducible factor-2alpha is a catabolic regulator of osteoarthritic cartilage destruction. Nat Med 16: 687-693.

Yoo JU, Barthel TS, Nishimura K, Solchaga L, Caplan AI, Goldberg VM, Johnstone B (1998) The chondrogenic potential of human bone-marrow-derived mesenchymal progenitor cells. J Bone Joint Surg Am 80: 1745-1757.

Yudoh K, Nakamura H, Masuko-Hongo K, Kato T, Nishioka K (2005) Catabolic stress induces expression of hypoxia-inducible factor (HIF)-1 alpha in articular chondrocytes: involvement of HIF-1 alpha in the pathogenesis of osteoarthritis. Arthritis Res Ther 7: R904914.
Zhou S, Cui Z, Urban JP (2004) Factors influencing the oxygen concentration gradient from the synovial surface of articular cartilage to the cartilage-bone interface: a modeling study. Arthritis Rheum 50: 3915-3924.

\section{Discussion with Reviewers}

Reviewer III: How do the authors envisage the use of predifferentiated MSCs in vivo?

Authors: While MSCs have been evaluated for their ability to aid in cartilage repair in a clinical setting using a variety of therapeutic approaches, the use of pre-differentiated MSCs has to-date been limited. In fact, preclinical studies in animals have suggested that pre-differentiation may be either beneficial (Zscharnack et al., 2010) or detrimental (Chang et al., 2011) compared with undifferentiated MSCs in MACI-type applications. With the tendency of MSCs to express high levels of matrix proteins not typically associated with hyaline cartilage (e.g. collagen types I and X), prospects for their use in such applications will most likely be limited unless the phenotype of the tissue produced can be better controlled. If pathways that direct these cells towards a hyaline cartilage chondrocyte phenotype can be defined, they could become a valuable cellular resource for in vivo applications, perhaps guided by innovative gene delivery technologies and supported by next-generation scaffolds. Breakthroughs in such technologies may one day allow for robust hyaline-like tissues to be synthesised from MSCs in a laboratory setting or, alternatively, to allow for improved control of in situ tissue development by these cells. Presently, their value as a precursor cell for cartilage repair is unclear.

\section{Additional References}

Zscharnack M, Hepp P, Richter R, Aigner T, Schulz R, Somerson J, Josten C, Bader A, Marquass B (2010) Repair of chronic osteochondral defects using predifferentiated mesenchymal stem cells in an ovine model. Am J Sports Med 38: 1857-1869.

Chang CH, Kuo TF, Lin FH, Wang JH, Hsu YM, Huang HT, Loo ST, Fang HW, Liu HC, Wang WC (2011) Tissue engineering-based cartilage repair with mesenchymal stem cells in a porcine model. J Orthop Res 29: 1874-1880.

Editor's Note: Scientific Editor in charge of the paper: Mauro Alini. 